\title{
Experimental evidence for inhomogeneous pumping and energy-dependent effects in photon Bose-Einstein condensation
}

\author{
J. Marelic and R. A. Nyman* \\ Centre for Cold Matter, Blackett Laboratory, Imperial College London, Prince Consort Road, London, SW7 2BW England, United Kingdom
}

(Received 24 October 2014; published 12 March 2015)

\begin{abstract}
Light thermalized at room temperature in an optically pumped, dye-filled microcavity resembles a model system of noninteracting Bose-Einstein condensation in the presence of dissipation. We have experimentally investigated some of the steady-state properties of this unusual state of light and found features which do not match the available theoretical descriptions. We have seen that the critical pump power for condensation depends on the pump beam geometry, being lower for smaller pump beams. Far below threshold, both intracavity photon number and thermalized photon cloud size depend on pump beam size, with optimal coupling when the pump beam matches the thermalized cloud size. We also note that the critical pump power for condensation depends on the cavity cutoff wavelength and longitudinal mode number, which suggests that energy-dependent thermalization and loss mechanisms are important.
\end{abstract}

DOI: 10.1103/PhysRevA.91.033813

PACS number(s): 42.50.Nn, 03.75.Hh, 67.10.Ba

The decision to categorize an experimentally observed phenomenon as Bose-Einstein condensation (BEC) goes hand in hand with the consensus microscopic description. For example, the popular definition of BEC by Penrose and Onsager [1] of extensive or macroscopic occupancy by identical bosons of a single quantum state was chosen to extend the original idea of Bose and Einstein to interacting particles, implicitly assuming homogeneity, in their case superfluid helium.

In general, BEC at thermal equilibrium arises because the Bose-Einstein distribution diverges when the chemical potential approaches the energy of the ground state (from below). In dissipative, nonequilibrium condensation of exciton polaritons in semiconductors (e.g., [2-4]) or of polaritons in organic molecules [5,6], the system may be effectively homogeneous, so the Penrose and Onsager definition of BEC applies, but thermal equilibrium is not always strongly established. In these cases, BEC is widely accepted when thermal equilibrium is experimentally demonstrated to be a good description, and a macroscopic population is observed in the lowest-energy state, despite the strong interactions.

Photons thermalized in a dye-filled microcavity probably have the weakest interactions of any system to have exhibited BEC, including atoms near Feshbach resonances [7,8]. In this intrinsically inhomogeneous system, thermal equilibrium and macroscopic occupancy of the ground state are the usual criteria for BEC, and both have been observed despite the dissipation $[9,10]$, so $\mathrm{BEC}$ is assigned without major controversy [11]. Interactions are so weak, that questions have been asked about the mechanism by which the condensate forms [12]. There has been considerable recent activity developing microscopic models of this physical system, but most of the models, e.g., by Kruchkov [13], assume that near-thermal-equilibrium conditions hold.

${ }^{*}$ Correspondence should be addressed to r.nyman@imperial.ac.uk

Published by the American Physical Society under the terms of the Creative Commons Attribution 3.0 License. Further distribution of this work must maintain attribution to the author(s) and the published article's title, journal citation, and DOI.
Using principals of detailed balance [14] and hierarchical maximum entropy $[15,16]$, fluctuations of the condensate population about the thermal equilibrium have been predicted and subsequently observed [17]. Likewise, low-energy excitations about the condensate mean-field such as the Bogoliubov dispersion [18,19] have also been calculated. Phase fluctuations can only be predicted by fully-quantized models including dissipation [20,21].

One published model $[22,23]$ looks at the limits of the thermalization process itself, and hence can state when BEC is and is not a good description. When thermalization is slower than loss, threshold may still be reached, but the macroscopically occupied mode may no longer be the ground state. In other words, BEC breaks down when thermalization breaks down. Validation of this and all the other models requires new experimental evidence.

We report here our own observations of dye-filled microcavity photon condensation in the steady state. We have seen that the critical pump power $P_{C}$ varies strongly with the pump beam geometry, in stark contrast to the predictions of a simple model assuming perfect equilibrium [10,13]. We demonstrate that even below threshold the equilibrium model is incomplete: the thermalized cloud size and photon number are also pump-geometry dependent. We also measure $P_{C}$ as ground-state energy and overall cavity length vary, and we explain our observations through energy-dependent losses. These steady-state features should be explained by any successful model of photon BEC.

We note that very recent experiments have looked at aspects of the time-resolved behavior of photon thermalization [24], and the crossover to lasing when thermalization fails.

Our experimental method is similar to that of Klaers et al. [9,10]. A fluorescent dye, rhodamine $6 \mathrm{G}$ dissolved in ethylene glycol at $2 \mathrm{mM}$ concentration, is held by surface tension between two dielectric mirrors placed 1-2 $\mu \mathrm{m}$ apart, as shown in Fig. 1 (top). One of the two mirrors is spherical with a radius of curvature $R=0.25 \mathrm{~m}$ and the other is planar, cut down to $1-\mathrm{mm}$ diameter. We pump the dye incoherently using $\lambda_{p}=532-\mathrm{nm}$ light, passing through the dielectric mirror at a transmission maximum angle around $37^{\circ}$ to the normal. To prevent shelving of the dye in the triplet state, 


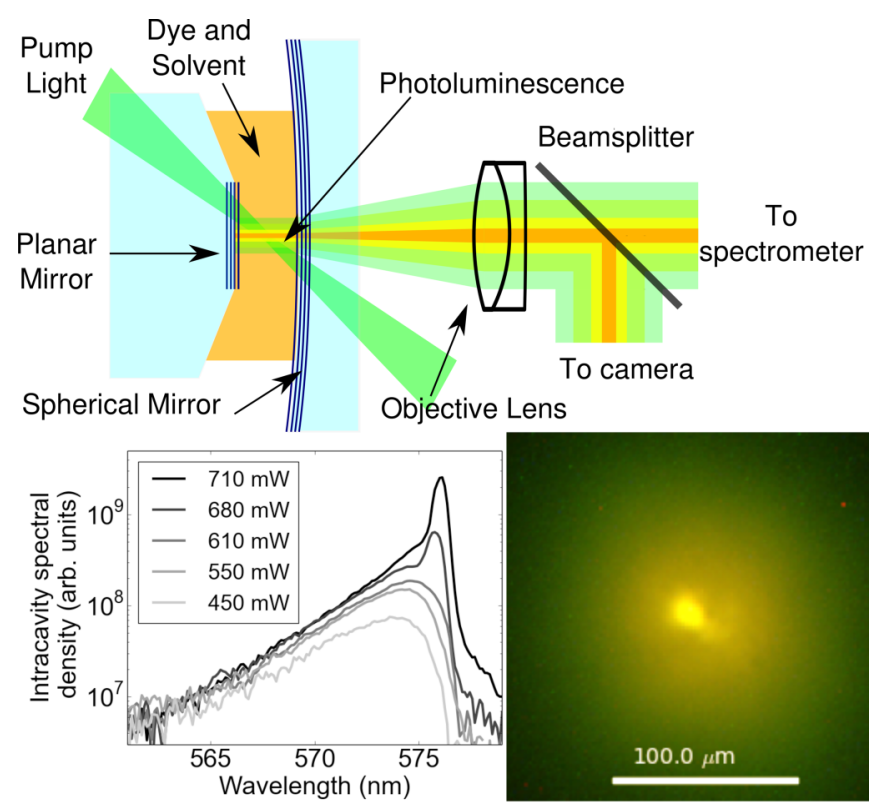

FIG. 1. (Color online) Schematic diagram of the apparatus (top) and some example data demonstrating BEC of photons in a dyefilled microcavity. Bottom left: spectra at varying intracavity pump powers, showing the saturation of the excited-state population and then condensation into the lowest-energy mode available. Bottom right: an image of a condensate just above threshold, in real colors, albeit with the intensity adjusted for visibility when printed.

we pulse the pump on for $500 \mathrm{~ns}$ at a repetition rate of $500 \mathrm{~Hz}$.

We collect the light leaking through the cavity mirrors, the photoluminescence, using a 50-mm focal length, $25-\mathrm{mm}$ diameter, achromatic doublet as an objective lens in an afocal setup: an image is effectively formed at infinity. The light is split and imaged onto a camera and a commercial spectrometer, whose entrance slit is easily replaceable. The length of the cavity is controlled using a piezoelectric actuator, and stabilized by reference to a collimated helium-neon laser (at $633 \mathrm{~nm}$ ) as observed by a secondary camera. Pump and stabilization wavelengths are separated from photoluminescence using a combination of dichroic, notch, and short-pass filters.

We show an example image of BEC of photons in Fig. 1 (bottom right). The colors correspond nearly to those observed by the camera. The thermal component is the broad Gaussian cloud around the condensate, which shows up as a bright central spot. In the spectrum, Fig. 1 (bottom left), the condensation shows up as an increase in the population of the lowest-energy state, at the cavity cutoff. The thermal component is compatible with a room-temperature thermal distribution, although here the $50-\mu \mathrm{m}$ spectrometer entrance slit cuts off some of the higher-energy components. The observed spectrum is divided by the known wavelength variation of the mirror transmission to obtain the intracavity spectrum.

We determine the threshold power using the deviation of the spatial variation of photoluminescence from a Gaussian near the center. This measure has proven to be precise and robust and is as well performed by eye as by any quantitative measure we have tried, e.g., output power as a function of pump power, or fitting of the spectra.
The simplest theory of thermalized BEC of dye-filled microcavity photons, as used in Ref. [10], assumes that a number of photons are trapped in the cavity at thermal equilibrium, and that above the critical number a condensate will form. The critical particle number per spin state for equilibrium Bose-Einstein condensation in a symmetric two-dimensional (2D) harmonic oscillator is $g\left(\pi^{2} / 6\right)\left(k_{B} T / \hbar \Omega\right)^{2}$ where $T$ is the temperature; $\Omega=\left(c / n_{L}\right) \sqrt{1 / L R}$ is the angular trapping frequency for photons in a cavity of length $L$, filled with a medium of refractive index $n_{L}$; and $g$ is the spin degeneracy.

The photon number stored in a dye-filled microcavity is equal to the light power absorbed from the pump times the time the light circulates for divided by the typical energy per photon absorbed: $N_{\text {cav }}=P_{p} n_{\text {mol }} A F \lambda_{0}^{2} \lambda_{p} q\left(q-q_{0}\right) / 2 n_{L} h c^{2}$. Here, $P_{p}$ is the pump light power, $n_{\mathrm{mol}}$ is the dye-molecule number density, and $A$ is the absorption cross section for light at $\lambda_{p}$. The lowest-energy mode of the cavity at the cutoff wavelength $\lambda_{0}$ is in the $q$ th longitudinal mode, $q_{0} \simeq 4$ indicates how far the light penetrates the surface of the mirrors, and $F \simeq$ 20000 is the typical number of round trips light will make in the cavity before decay, finesse divided by $\pi$. The critical pump power is then $P_{C}=2 \pi^{4} g R\left(k_{B} T n_{L}\right)^{2} / 3 h n_{\mathrm{mol}} A F \lambda_{p} \lambda_{0}(q-$ $q_{0}$ ). We note that the predictions of this model are independent of the spatial distribution of pump light. It is assumed that all the pump light absorbed is fully thermalized, and a single lifetime is assigned to all cavity modes at all energies. The model also ignores the effects of photon reabsorption by dye molecules.

The pump light is focused into the cavity through the planar mirror. To measure the pump spot size, $\sigma_{p}$, we observe the photoluminescence from a cavity around $30 \mu \mathrm{m}$ long, where thermalization breaks down. We fit a 2D Gaussian ellipse and infer two width parameters (root mean square). We give uncertainties in $\sigma_{p}$ which are half the difference between the two width parameters.

The theory we have presented assumes that how photons are pumped into the cavity is irrelevant, so $P_{C}$ should be independent of pump geometry. In Fig. 2 we present experimental observations of $P_{C}$ as a function of pump spot

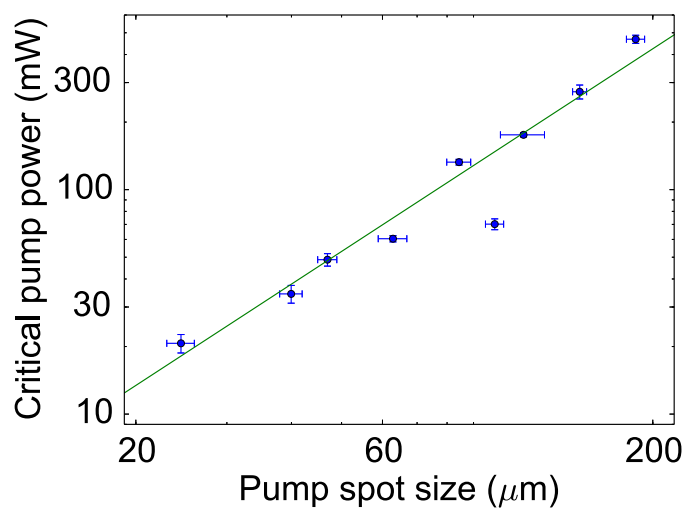

FIG. 2. (Color online) Critical intracavity pump power variation with pump spot size $\sigma_{p}$ for the eighth longitudinal mode and cavity cutoff wavelength $586 \mathrm{~nm}$. The line is a guide to the eye, proportional to $\sigma_{p}^{1.5}$. Error bars are from the differences in inferred size of the two principal axes of a two-dimensional Gaussian fitted to the pump spot images. 
size which directly contradict the prediction of our simple model, which gives around $1 \mathrm{~mW}$ for these parameters, far below the measured values. The data can be fitted well to a power law, $P_{C} \propto \sigma_{p}^{1.5 \pm 0.1}$, although there is no reason to believe that the power law extends beyond the range of our measurements. The critical pump intensity decreases with increasing pump spot size.

There are two main saturation mechanisms in dye fluorescence, stimulated emission and pumping into a dark state, neither of which can explain our observations. The stimulated-emission saturation intensity for absorption at our pump wavelength is approximately $I_{\mathrm{sat}}=h c / \lambda_{p} A \tau_{\mathrm{sp}}=$ $4 \times 10^{9} \mathrm{~W} \mathrm{~m}^{-2}$, taking the spontaneous emission lifetime as $\tau_{\mathrm{sp}}=4 \mathrm{~ns}[25,26]$. The highest intensity we find at threshold is $6 \times 10^{6} \mathrm{~W} \mathrm{~m}^{-2}$; stimulated emission is a negligible effect. The rate of nonradiative events, mostly intersystem crossing into the triplet state, is found from the fluorescence quantum yield, $\Phi \simeq 95 \%$ [27]. The typical time scale for these events to occur with our pump intensities is $\tau_{S T}=\tau_{\mathrm{sp}} \frac{I_{\mathrm{sat}}}{I(1-\Phi)}>50 \mu \mathrm{s}$, much longer than our pulses. Recovery from the triplet state is no slower than $400 \mu \mathrm{s}$ [28], so all molecules return to the singlet state between pulses.

Since saturation mechanisms are not responsible, we suppose that spatial redistribution of photons from the pump to the thermalized distribution is not fully efficient. It is worth noting that the smallest pump spots used in any of our experiments, $15 \mu \mathrm{m}$, are not much larger than the smallest cavity mode, about $6 \mu \mathrm{m}$. Where threshold behavior is seen, independent of the pump geometry, it is always the lowest-energy transverse mode which is macroscopically occupied. Along with the thermal excitations seen in both the images and the spectra, this feature points to BEC being a good description $[10,11,22]$.

We now turn to below-threshold thermalization behavior, where we have observed both the number of thermalized photons $N_{\text {cav }}$ and their spatial distribution as $\sigma_{p}$ varies. We infer $N_{\text {cav }}$ by measuring the output light power $P_{m}$ and accounting for the mirror transmission $T_{M}(\lambda)$, a slowly varying function of wavelength $\lambda$, and cavity round-trip time: $N_{\text {cav }}=$ $P_{m} q \lambda_{0}^{2} / h c^{2} T_{M}\left(\lambda_{0}\right)$.

There are no predictions for how the inhomogeneous pump beam generates a thermalized population of intracavity photons. Our observations in Fig. 3 indicate variations in coupling efficiency up to a factor of about 2 , although always much lower efficiency than our simple model, which predicts about 20000 photons per $\mathrm{mW}$ of pump. The observations are for continuous-wave (CW) pumping, always less than $20 \%$ of critical power, observing no power-dependent effects over a factor of 15 in pump power, ruling out saturation phenomena. We note that for every free photon there are approximately $A\left(\lambda_{0}\right) c \tau_{\mathrm{sp}} n_{\mathrm{mol}} / 4 n_{L}$ molecules in the excited state, where $A\left(\lambda_{0}\right) \propto \exp \left(-h c / \lambda_{0} k_{B} T\right)$ is the absorption cross section of the molecules at the cutoff wavelength (for large detuning from the molecular resonance) [29]. This correction to our model is wavelength dependent and for the parameters of Fig. 3 is about 2.9, i.e., a significant correction, but not enough to explain the discrepancy.

We have measured the transmission of the pump light as a function of cavity length, and inferred a concentration of $4.8 \mathrm{mM}$ of optically active dye molecules under typical experimental conditions. Photobleaching, which causes

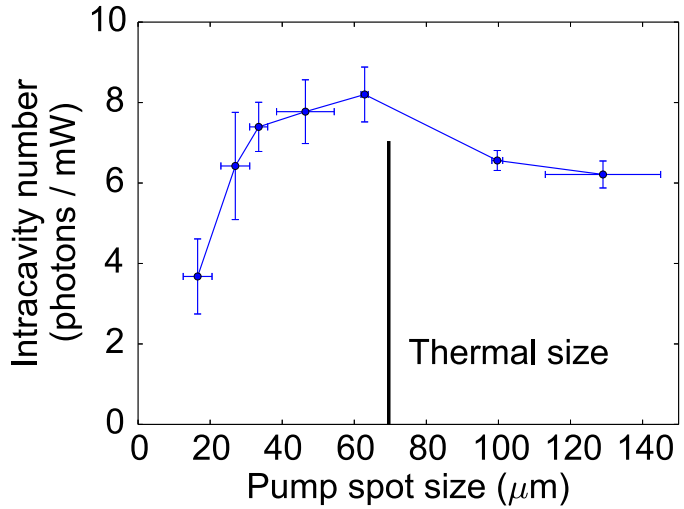

FIG. 3. (Color online) Cavity photon number normalized to intracavity CW pump power well below threshold, as pump spot size varies, for the eighth longitudinal mode with $\lambda_{0}=590 \mathrm{~nm}$. Horizontal error bars are from ellipticity of a 2D Gaussian fit. Vertical error bars are from variation among three series of measurements spanning a factor 15 in pump power.

molecules permanently to stop fluorescing, can be ruled out as a consequence. The increase in concentration is due to solvent evaporation over the several days between dye changes: the measurement was performed five days after the last dye change, after two working days of data taking.

The thermal cloud, below threshold, is expected to have a root-mean-square size of $\sigma_{\text {th }}=\sqrt{k_{B} T q \lambda_{0}^{2} R / 2 h c n_{L}}$. The optimal coupling between pump and thermalized light is found when the pump and thermal spatial distributions match, $\sigma_{p}=$ $\sigma_{\text {th }}$. A possible explanation lies in the fact that the pump excites molecules preferentially near the cavity center, more markedly so for smaller pump spots. Redistribution of the emitted light by multiple scattering from molecules is not fully efficient. It is possible for large $\sigma_{p}$ that some of pump light strikes damaged regions of the mirrors. Combined with the results of Fig. 2, we conclude that the threshold photon number decreases rapidly for decreasing pump spot size below the typical thermal cloud size, faster than linearly.

We use a second measure of thermalization, the photon cloud size $\sigma_{c}$, by fitting a 2D Gaussian to the photoluminescence. Uncertainties in the data are again from the ellipticity of the fit. In Fig. 4 we see the photon cloud size falls between pump spot size and thermal size. For larger $\sigma_{p}$ we find $\sigma_{c} \simeq \sigma_{\text {th }}$, but not for small $\sigma_{p}$. The implication is that light from larger pump spots thermalizes better than small spots, but that pumping with a spot the same size as the thermal cloud gives optimized coupling.

Thermalization requires the photon-dye scattering rate to be faster than the cavity loss rate, and both rates are wavelength dependent. The scattering rate decreases exponentially with increasing wavelength, and our cavity mirrors have maximum reflectivity at about $550 \mathrm{~nm}$, so thermalization breaks down at longer wavelengths. We have made below-threshold cloud-size measurements in the range $\lambda_{0}=575-610 \mathrm{~nm}$ at concentrations from 0.02 to $2 \mathrm{mM}$. The rate of scattering, hence the thermalization rate, varies by a factor of 20000 . For small $\sigma_{p}$ we see no systematic variation in $\sigma_{c}$ with scattering rate. For large $\sigma_{p}$ there is some evidence that higher scattering rates 


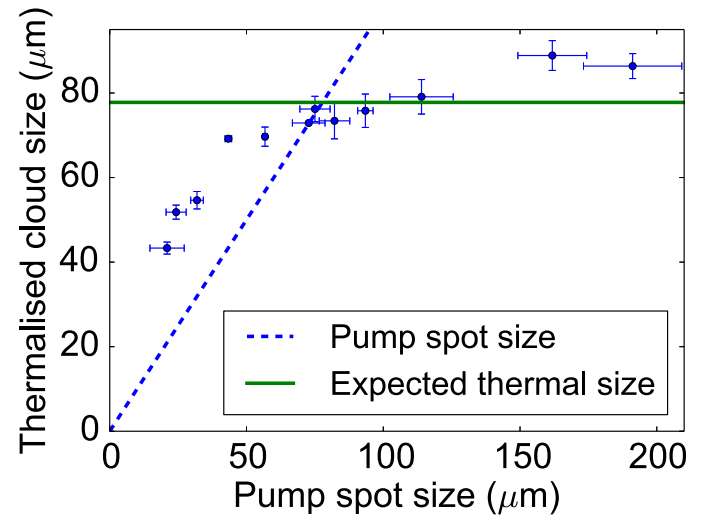

FIG. 4. (Color online) Thermalized photon cloud size variation with pump spot size for CW pump well below threshold. For comparison, the expected thermal cloud size for room temperature is plotted, alongside a line equal to the pump spot size. Error bars are from the differences in inferred size of the two principal axes of a two-dimensional Gaussian fitted to the images. The thermalized cloud is observed in the tenth longitudinal mode with cavity cutoff $590 \mathrm{~nm}$.

are associated with cloud sizes that better match the expected thermal cloud size.

Threshold observations, as in Fig. 5, reveal that for each longitudinal mode number $q P_{C}$ for BEC shows a minimum as a function of the cavity cutoff wavelength. Cavity cutoff $\lambda_{0}$ is determined by the peak emission wavelength above threshold, i.e., the BEC wavelength. There are predictions that $P_{C}$ decreases exponentially with increasing wavelength, either with an offset [13] or without [22], because, close to the molecular resonance (short wavelengths), excitations are attached to dye molecules and it is only the free photons that are involved in the BEC. Going to longer $\lambda_{0}$, when the cavity loss rate becomes comparable to the thermalization rate, thermalization breaks down and the $P_{C}$ increases [23]. Nonradiative scattering, which occurs about once for every $1 /(1-\Phi) \simeq 20$ radiative thermalizing scattering events, becomes an important loss mechanism at short $\lambda_{0}$. Since the scattering rate varies by that factor every $9 \mathrm{~nm}$ or so, $P_{C}$ varies on this scale.

At longer cavity lengths, larger $q$, the lowest $P_{C}$ shifts to longer wavelengths. For longer cavities, the photons meet the mirrors less frequently, hence the cavity loss rate is lower, so the balance between loss mechanisms shifts to longer wavelengths, where scattering events are more infrequent.

In conclusion, we have observed dye-filled microcavity photon BEC, and seen that the macroscopic occupation of

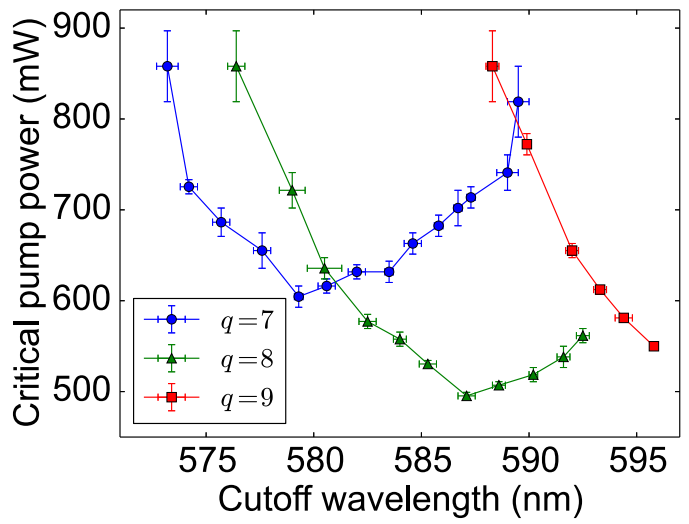

FIG. 5. (Color online) Critical power $P_{C}$ against cavity cutoff wavelength $\lambda_{0}$ (the wavelength at which BEC appears), for various longitudinal mode numbers, $q$, suggestive of energy-dependent loss mechanisms. $\sigma_{p}=170 \mu \mathrm{m}$. Error bars come from uncertainty in determining threshold and from the observed wavelength jitter during experiments.

the lowest-energy state is a robust phenomenon. We have noted behavior which was dependent on both the pump beam geometry and the cutoff wavelength of the cavity. Our observations are not in contradiction to those of Refs. [9,10,24] using off-centered pump beams. The critical pump power increases faster than linearly with pump spot size over the range that we have measured. The efficiency of coupling from pump light to intracavity photon number also increases with spot size, for spots smaller than the typical thermal size, implying that critical photon number increases dramatically. The size of the intracavity photon cloud also depends on pump spot size. This evidence suggests that the pump excites molecules which emit into modes which approximate the pump spot shape, and that the spatial redistribution of light is not complete. We have also observed that critical pump power depends on ground-state energy, with an optimum dictated by a balance between loss mechanisms: cavity photon loss and nonradiative photon scattering by the dye. We believe a model of dye-filled microcavity photon BEC that included these effects would be able to fully explain our results, and that the inclusion of these effects would render predictions of phenomena such as coherence and fluctuations more accurate.

We thank Peter Kirton, Jonathan Keeling, and Jan Klaers for many informative discussions and Lydia Zajiczek for experimental assistance. This work was funded by the EPSRC (United Kingdom).
[1] O. Penrose and L. Onsager, Phys. Rev. 104, 576 (1956)

[2] J. Kasprzak, M. Richard, S. Kundermann, A. Baas, P. Jeambrun, J. M. J. Keeling, F. M. Marchetti, M. H. Szymańska, R. André, J. L. Staehli, V. Savona, P. B. Littlewood, B. Deveaud, and Le Si Dang, Nature (London) 443, 409 (2006).

[3] H. Deng, D. Press, S. Götzinger, G. S. Solomon, R. Hey, K. H. Ploog, and Y. Yamamoto, Phys. Rev. Lett. 97, 146402 (2006).

[4] R. Balili, V. Hartwell, D. Snoke, L. Pfeiffer, and K. West, Science 316, 1007 (2007).
[5] K. Daskalakis, S. Maier, R. Murray, and S. Kéna-Cohen, Nat. Mater. 13, 271 (2014).

[6] J. D. Plumhof, T. Stöferle, L. Mai, U. Scherf, and R. F. Mahrt, Nat. Mater. 13, 247 (2014).

[7] P. Krüger, Z. Hadzibabic, and J. Dalibard, Phys. Rev. Lett. 99, 040402 (2007).

[8] M. Gustavsson, E. Haller, M. J. Mark, J. G. Danzl, G. RojasKopeinig, and H.-C. Nägerl, Phys. Rev. Lett. 100, 080404 (2008). 
[9] J. Klaers, F. Vewinger, and M. Weitz, Nat. Phys. 6, 512 (2010).

[10] J. Klaers, J. Schmitt, F. Vewinger, and M. Weitz, Nature (London) 468, 545 (2010).

[11] B. Fischer and R. Weill, Opt. Express 20, 26704 (2012).

[12] D. Snoke and S. Girvin, J. Low Temp. Phys. 171, 1 (2013).

[13] A. Kruchkov, Phys. Rev. A 89, 033862 (2014).

[14] J. Klaers, J. Schmitt, T. Damm, F. Vewinger, and M. Weitz, Phys. Rev. Lett. 108, 160403 (2012).

[15] D. N. Sob'yanin, Phys. Rev. E 85, 061120 (2012).

[16] D. N. Sob'yanin, Phys. Rev. E 88, 022132 (2013).

[17] J. Schmitt, T. Damm, D. Dung, F. Vewinger, J. Klaers, and M. Weitz, Phys. Rev. Lett. 112, 030401 (2014).

[18] J.-J. Zhang, J.-H. Yuan, J.-P. Zhang, and Z. Cheng, Physica E 45, 177 (2012).

[19] R. A. Nyman and M. H. Szymańska, Phys. Rev. A 89, 033844 (2014).

[20] A.-W. de Leeuw, H. T. C. Stoof, and R. A. Duine, Phys. Rev. A 88, 033829 (2013).

[21] A. Chiocchetta and I. Carusotto, Phys. Rev. A 90, 023633 (2014).
[22] P. Kirton and J. Keeling, Phys. Rev. Lett. 111, 100404 (2013).

[23] P. Kirton and J. Keeling, arXiv:1410.6632.

[24] J. Schmitt, T. Damm, D. Dung, F. Vewinger, J. Klaers, and M. Weitz, arXiv:1410.5713.

[25] D. Magde, G. E. Rojas, and P. G. Seybold, Photochem. Photobiol. 70, 737 (1999).

[26] Dye Lasers, edited by F. Schaefer (Springer-Verlag, Berlin, 1990).

[27] D. Magde, R. Wong, and P. G. Seybold, Photochem. Photobiol. 75, 327 (2002).

[28] R. Zondervan, F. Kulzer, S. B. Orlinskii, and M. Orrit, J. Phys. Chem. A 107, 6770 (2003).

[29] We have measured the absorption scattering cross section using a spectrophotometer. Our measurements match well with $A\left(\lambda_{0}\right) \propto$ $\exp \left(-h c / \lambda_{0} k_{B} T\right)$ in the range $565 \mathrm{~nm}$ to at least $620 \mathrm{~nm}$, as well as being compatible with the available literature nearer to resonance [30], with $A(590 \mathrm{~nm})=1.2 \times 10^{-23} \mathrm{~m}^{2}$.

[30] P. Bojarski, A. Matczuk, C. Bojarski, A. Kawski, B. Kukliski, G. Zurkowska, and H. Diehl, Chem. Phys. 210, 485 (1996). 